\title{
Performance of the STACEE Atmospheric Cherenkov Telescope
}

\author{
D.A. Williams, ${ }^{1}$ D. Bhattacharya,${ }^{2}$ L.M. Boone, ${ }^{1}$ M.C. Chantell, ${ }^{3}$ \\ Z. Conner, ${ }^{3 *}$ C.E. Covault,${ }^{3}$ M. Dragovan,${ }^{3 \dagger}$ P. Fortin, ${ }^{4}$ D. Gingrich, ${ }^{5}$ \\ D.T. Gregorich, ${ }^{6}$ D.S. Hanna, ${ }^{4}$ G. Mohanty, ${ }^{2}$ R. Mukherjee, ${ }^{7}$ \\ R.A. Ong, ${ }^{3}$ S. Oser, ${ }^{3 \ddagger}$ K. Ragan, ${ }^{4}$ R.A. Scalzo, ${ }^{3}$ D.R. Schuette, ${ }^{3}$ \\ C.G. Théoret, ${ }^{4}$ T.O. Tümer, ${ }^{2}$ F. Vincent,${ }^{4}$ J.A. Zweerink ${ }^{2}$
}

\footnotetext{
${ }^{1}$ Santa Cruz Institute for Particle Physics, Univ. of California, Santa Cruz, CA 95064, USA

${ }^{2}$ Institute of Geophysics and Planetary Physics, Univ. of California, Riverside, CA 92521, USA

${ }^{3}$ Enrico Fermi Institute, Univ. of Chicago, Chicago, IL 60637, USA

${ }^{4}$ Dept. of Physics, McGill Univ., Montreal, Quebec H3A 2T8, Canada

${ }^{5}$ Centre for Subatomic Research, Univ. of Alberta, Edmonton, Alberta T6G 2N5, Canada

${ }^{6}$ Dept. of Physics 8 Astronomy, California State Univ., Los Angeles, CA 90032, USA

${ }^{7}$ Dept. of Physics $\&$ Astronomy, Barnard Coll. $\&$ Columbia Univ., New York, NY 10027, USA

*Present address: Dept. of Physics, George Washington Univ., Washington, D.C. 2007r, USA

${ }^{\dagger}$ Present address: Jet Propulsion Laboratory, Pasadena, CA 91109, USA

$\ddagger$ Present address: Dept. of Physics $\&$ Astronomy, Univ. of Pennsylvania, Philadelphia, PA 19104, USA
}

\begin{abstract}
The Solar Tower Atmospheric Cherenkov Effect Experiment (STACEE) is located at the National Solar Thermal Test Facility of Sandia National Laboratories in Albuquerque, New Mexico, USA. The field of solar tracking mirrors (heliostats) around a central receiver tower is used to direct Cherenkov light from atmospheric showers onto secondary mirrors on the tower, which in turn image the light onto cameras of photomultiplier tubes. The STACEE Collaboration has previously reported a detection of the Crab Nebula with approximately 7 standard deviation significance, using 32 heliostats (STACEE-32). This result demonstrates both the viability of the technique and the suitability of the site. We are in the process of completing an upgrade to 48 heliostats (STACEE-48) en route to an eventual configuration using 64 heliostats (STACEE-64) in early 2001. In this paper, we summarize the results obtained on the sensitivity of STACEE-32 and our expectations for STACEE-48 and STACEE-64.
\end{abstract}

Astrophysical measurements in the 10 to $300 \mathrm{GeV}$ region of the gamma-ray spectrum have proved elusive to both ground-based and satellite techniques. Satellite detectors have been too small to detect the faint high-energy fluxes. Ground-based detectors can achieve large collection areas, but rely on detecting the secondary products of extensive air showers in the atmosphere. The imaging atmospheric 
Cherenkov technique has been quite successful above a few hundred GeV [1], the lowest threshold at which the Cherenkov light image stands out above the night-sky background. Builders of the next generation of imaging telescopes aspire to thresholds as low as $10 \mathrm{GeV}$ [2]. STACEE is a wavefront-sampling Cherenkov telescope which, by using an existing solar facility, has a large mirror area to achieve a low threshold and has been implemented in a comparatively short time.

STACEE is situated at the National Solar Thermal Test Facility $\left(34.962^{\circ} \mathrm{N}\right.$, $106.509^{\circ} \mathrm{W}, 1705 \mathrm{~m}$ above sea level) near Albuquerque, New Mexico. The facility has 212 heliostat mirrors, each about $37 \mathrm{~m}^{2}$ area, designed to track the Sun and direct the image onto a central tower. As part of the STACEE design, we performed a detailed evaluation of the suitability of the site and the infrastructure. The results have been summarized previously [3]. For STACEE, the heliostats are used at night to direct Cherenkov light from gamma-ray showers in the atmosphere onto secondary mirrors on the central tower. The secondaries focus the light onto cameras of photomultiplier tubes. The secondary mirrors, phototube cameras, trigger and read-out electronics, and data acquisition system must be provided by the STACEE group, as well as software for retargeting the heliostats. The heliostats and their control system are maintained by the staff of the Test Facility.

We are working towards instrumenting 64 of the heliostats for use in STACEE. Since the time of flight for light from each heliostat to the secondary on the tower is different, the light from each heliostat must be focussed on a separate phototube in the camera in order to preserve the timing information. Once we had completed the instrumentation of 32 heliostats ("STACEE-32"), we took a substantial data set on the Crab nebula and pulsar. We presently have 48 heliostats instrumented ("STACEE-48"), and expect to complete the preparation of the final 16 to complete STACEE-64 in early 2001. The results from the analysis of the STACEE-32 Crab data $[4,5]$ provide the most comprehensive information we have so far on STACEE performance. We will first summarize those results. We refer the reader to the references for the full details. We will then discuss our expectations for STACEE48 and STACEE-64.

STACEE-32 was used to collect data from the Crab from November 1998 to February 1999. In addition to any possible gamma-ray signal from the Crab, there is a large isotropic background of showers from charged cosmic rays, especially protons. To account for this background, we compare the event rate tracking the direction of the Crab ("on-source") to the rate tracking the same path on the sky in local coordinates, $7.5^{\circ}$ to the east or west of the Crab position in right ascension ("off-source"). Data were collected in one hour cycles, consisting of two 28 minute runs (one on-source and one off-source) with 2 minutes between runs for slewing to the new position. Weather conditions were closely monitored, and only on/off pairs during good, stable conditions were used in the analysis. In some cases a questionable period was identified in one run of a pair. That period and the corresponding period in the second run of the pair were both removed, in order to have matched exposure in the on and off data sets. Events collected during periods satisfying the selection criteria for good conditions show a 5.3 standard deviation 


\begin{tabular}{rcccc}
\hline \hline Quantity & Nov 98 & Dec 98 & Jan/Feb 99 & Total \\
\hline On-Source Time $(\mathrm{s})$ & 56056 & 51239 & 48040 & 155335 \\
On-Source Events & 76235 & 55634 & 51046 & 182915 \\
Off-Source Events & 74686 & 54342 & 49825 & 178853 \\
Significance & $4.0 \sigma$ & $3.9 \sigma$ & $3.8 \sigma$ & $6.8 \sigma$ \\
Excess Rate $\left(\min ^{-1}\right)$ & $1.7 \pm 0.4$ & $1.5 \pm 0.4$ & $1.5 \pm 0.4$ & $1.57 \pm 0.23$ \\
\hline \hline
\end{tabular}

TABLE 1. Event excesses from the direction of the Crab. The January and February data have been combined, because fewer runs were taken in those months.

excess in the on-source events.

All of these events were then reconstructed using the information on the time of arrival of the Cherenkov light pulse at each heliostat. Some events that result from night-sky background fluctuations can be eliminated by reimposing the trigger condition in software. The 32 heliostats are organized into clusters of 8 . In order to trigger, there must be at least 3 of the 4 clusters which have 5 or more hits each. This condition is implemented in hardware using programmable delays and coincidence modules. It can be replicated somewhat more precisely in software using the recorded hit times.

The Cherenkov wavefront for a $\approx 100 \mathrm{GeV}$ gamma-ray shower can be approximated as a sphere centered at the shower maximum. The wavefront for protoninitiated showers is much more irregular. We use this as a means of preferentially selecting gamma-ray showers and suppressing the background. We fit the timing information for each shower to a sphere, and keep only those events for which the $\chi^{2}$ per degree of freedom is less than 1.0. The results for the number of events passing these requirements are summarized in Table 1. The excess has been enhanced to 6.8 standard deviations by the imposition of the timing requirements, an effect which would be expected if the excess is the result of a gamma-ray signal. The excess rate is steady throughout the data set, which supports the premise of a steady signal.

In order to determine the flux corresponding to the observed excess, we have done a thorough study of the STACEE-32 performance. We have used a CCD camera to record images of the Sun on the front face of the solar tower and images of the full Moon in the focal plane of the PMT camera. These images have been used to evaluate the collection efficiency of the optics. We have made a detailed simulation of the electronics and trigger, including the measured shape and variety of single photoelectron pulses and the effects of night-sky background light. We use the program MOCCA to do a full simulation of the shower development and Cherenkov light production in the atmosphere. The effective area that we obtain for STACEE-32, averaged over our Crab exposure, is shown in Fig. 1. Multiplied by a differential source spectrum proportional to $E^{-2.4}$, we obtain a spectral energy 

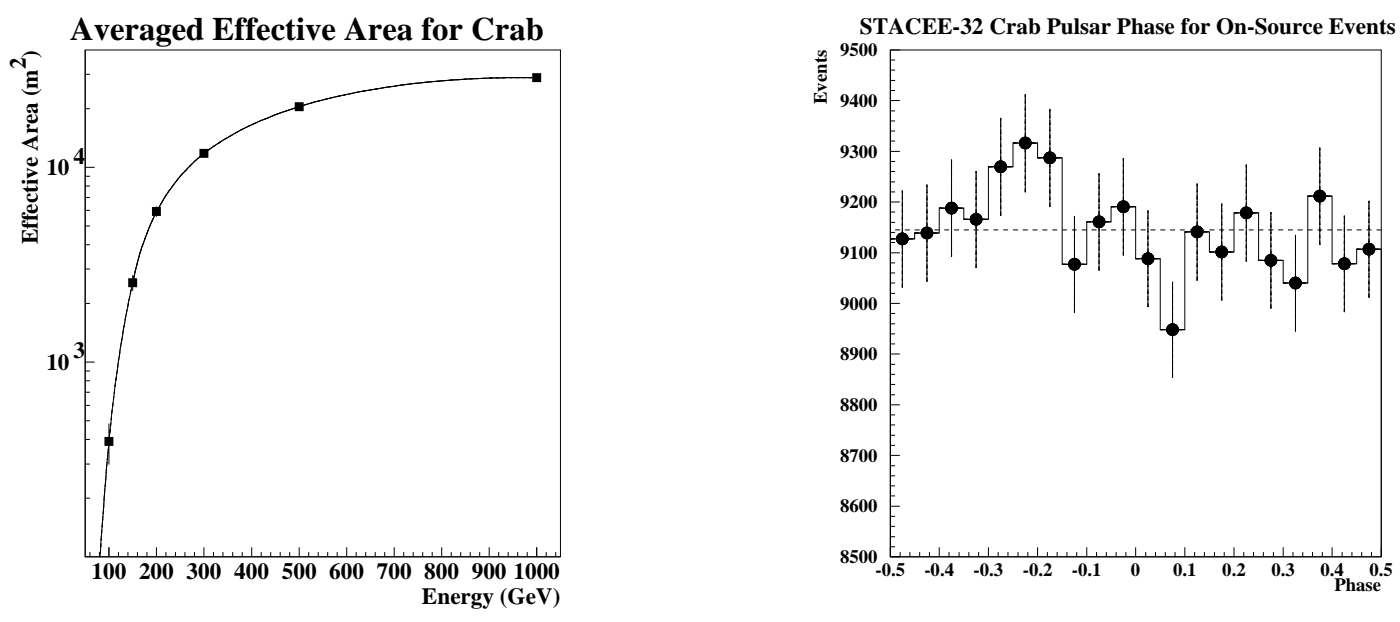

FIGURE 1. On the left, the Effective Area for STACEE-32. On the right, the STACEE-32 Crab Pulsar Phase Histogram. (C)2001. The American Astronomical Society. [5])

threshold, $E_{t h}$ (defined as the peak of the observed differential spectrum) of $190 \pm 60$ $\mathrm{GeV}$. We measure the integral flux of gamma rays from the Crab Nebula above $E_{t h}$ to be

$$
I\left(E>E_{t h}\right)=(2.2 \pm 0.6 \pm 0.2) \times 10^{-10} \text { photons } \mathrm{cm}^{-2} \mathrm{~s}^{-1} .
$$

The first error is statistical, and the second error is the systematic error on the flux itself, not including the effects of uncertainty in the energy threshold. The uncertainty in $E_{t h}$ does not change the flux value itself, but reflects the uncertainty in the energy above which the flux is integrated.

We have searched for evidence of pulsed emission in our detected excess from the Crab. The phase distribution of on-source events is shown in Fig. 1, where 0.0 corresponds to the phase of the main radio pulse. The distribution is consistent with being flat, and we place an upper limit on the fraction of the excess in the phase intervals where EGRET [6] has observed pulsed emission at lower energy. Those intervals are $0.94-0.04$ and $0.32-0.43$. The STACEE -32 limit on the pulsed fraction in these intervals is $<5.5 \%$ at the $90 \%$ confidence level.

Based on our work with STACEE-32, we can begin to extrapolate to the performance of STACEE-48 and STACEE-64. The most obvious upgrade is the addition of more heliostats, improving the light collection and the ability to pick out faint flashes. We are making several other improvements "behind the scenes" as well:

- We are adding $1 \mathrm{GHz}$ Flash ADC's on all channels for better time and pulse amplitude resolution, especially for small pulses.

- We have a new trigger with a tighter coincidence window (presently $8 \mathrm{~ns}$, but capable of being reprogrammed even tighter) with lower dead time at high rates. 
- We have improved the focusing and alignment of the heliostats to increase the amount of light reaching the secondary mirrors.

- We have applied a black sealant to the ground underneath the heliostats to reduce the albedo.

We are still in the process of evaluating the improvement in sensitivity from all of these changes. We expect to achieve a spectral energy threshold below $100 \mathrm{GeV}$

for STACEE-48, and near $50 \mathrm{GeV}$ for STACEE-64, with much increased effective area compared to STACEE-32 in both cases.

This work was supported in part by the National Science Foundation, the Natural Sciences and Engineering Research Council, Fonds pour la Formation de Chercheurs et l'Aide à la Recherche, the Research Corporation, and the California Space Institute.

\section{REFERENCES}

1. Ong, R.A., Phys. Rep. 305, 93 (1998); Hoffman, C.M. et al., Rev. Mod. Phys. 71, 897 (1999); Catanese, M. and Weekes, T.C., Proc. Astron. Soc. Pacific 111, 1193 (1999).

2. Lorenz, E., in de Jager, O.C., ed., Towards a Major Atmospheric Cerenkov Detector - V, Potchefstroom: Space Research Unit, 1997, pp. 415-421; Weekes, T.C. et al., ibid., pp. 433-446; Hofmann, W., ibid., pp. 405-414.

3. Chantell, M.C. et al., Nucl. Instrum. Meth. Phys. Res. A 408, 468 (1998).

4. Oser, S.M., University of Chicago, Department of Physics, Ph.D. Thesis (2000).

5. Oser, S. et al., to be published in Astrophys. J., astro-ph/0006304.

6. Nolan, P.L. et al., Astrophys. J. 409, 697 (1993); Ramanamurthy, P.V. et al., Astrophys. J. 470, 469 (1995); Fierro, J.M. et al., Astrophys. J. 494, 734 (1998). 